Supporting Information

\title{
Green Synthesis of Thin-Film Composite Membranes for Organic Solvent Nanofiltration
}

Chisiang Ong ${ }^{a}$, Gheorghe Falca ${ }^{a}$, Tiefan Huang ${ }^{a}$, Jiangtao Liu ${ }^{a}$, Priyanka Manchanda ${ }^{a}$, Stefan Chisca $^{a}$, Suzana P. Nunes ${ }^{a *}$

${ }^{a}$ King Abdullah University of Science and Technology (KAUST), Biological and Environmental Science and Engineering Division, Advanced Membranes and Porous Materials Center, 239556900 Thuwal, Saudi Arabia

*Corresponding author: Suzana P. Nunes, email suzana.nunes@kaust.edu.sa

13 Pages, 6 Figures and 5 Tables 


\section{List of figures and tables}

Figure S1. Contact angle measurement of (i) PAN porous support and (ii, iii, iv) polyamidePAN membranes prepared with $0.05 \%, 0.1 \%$ and $0.15 \%$ TMC in decanoic acid as organic phase.

Figure S2. UV spectra of dye solutions in methanol, before and after filtration, using a polyamide-PAN membrane (0.05\% TMC): (a) Methyl Orange, (b) Orange G, (c) Congo Red, and (d) Rose Bengal.

Figure S3. UV spectra of dye solutions in methanol, before and after filtration, using a polyamidePAN membrane (0.1\% TMC): (a) Methyl Orange, (b) Orange G, (c) Congo Red, and (d) Rose Bengal.

Figure S4. UV spectra of dye solutions in methanol, before and after filtration, using a polyamidePAN membrane (0.15\% TMC): (a) Methyl Orange, (b) Orange G, (c) Congo Red, and (d) Rose Bengal.

Figure S5. Zeta potential of (a) Methyl Orange (b) Orange G (c) Congo Red (d) Rose Bengal measured in solution in methanol.

Figure S6. Long term filtration tests: (a) methanol permeance and Congo Red rejection; (b) water permeance, using a polyamide-PAN membrane $(0.15 \% \mathrm{TMC})$.

Table S1. Types of dyes used and their properties.

Table S2. Types of solvent used and their properties.

Table S3. Mean pore diameter of the polyamide-PAN membranes with different TMC concentrations.

Table S4. Effect of TMC concentrations to the membrane zeta potential.

Table S5. Solvent stability test for a polyamide-PAN membrane $(0.1 \% \mathrm{TMC})$ in various solvents for 7 days. 
Table S1. Types of dyes used and their properties.

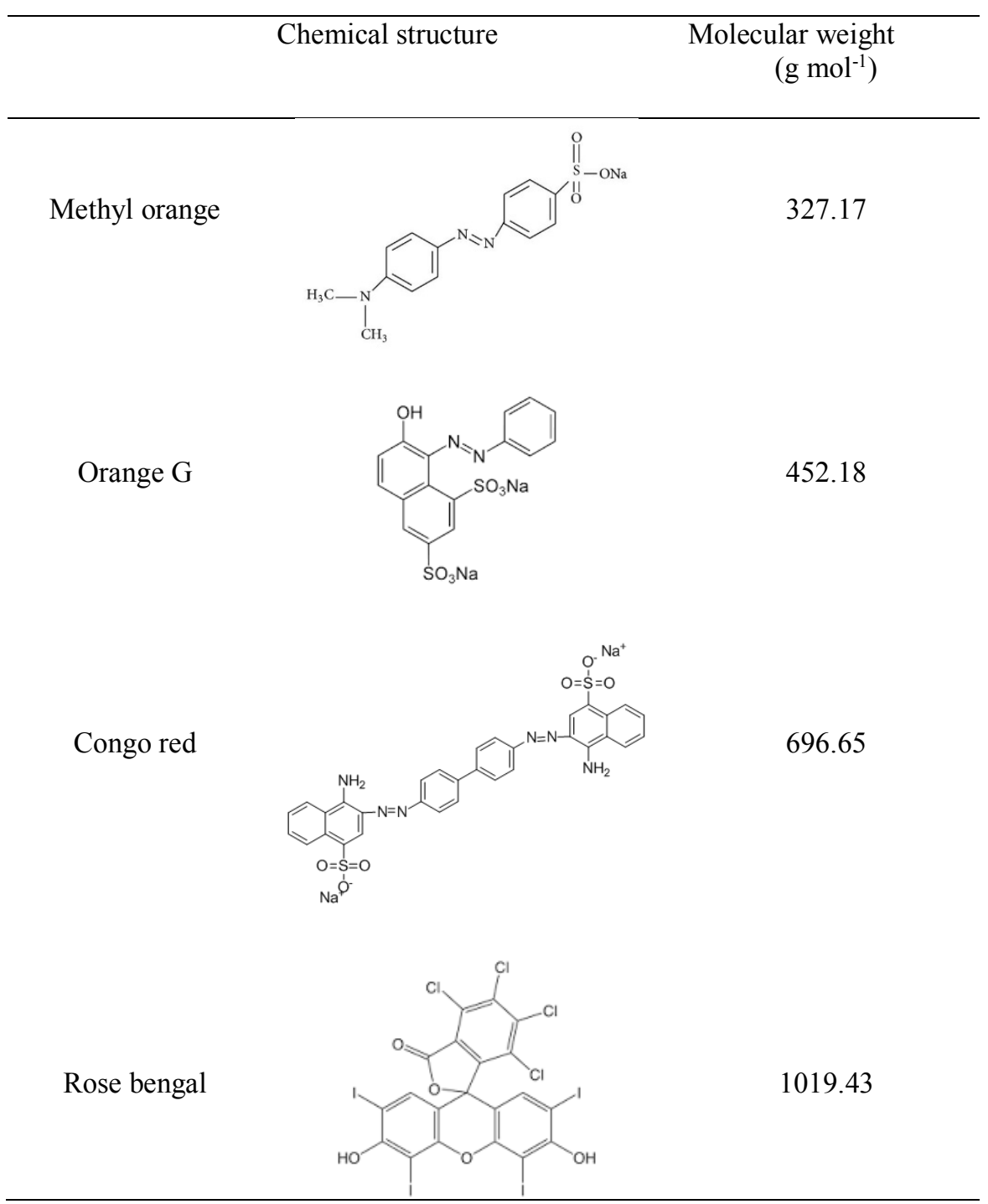


Table S2. Types of solvents used and their properties*

\begin{tabular}{|c|c|c|c|c|c|c|c|c|c|}
\hline & $\begin{array}{l}\text { Molecular } \\
\text { formula }\end{array}$ & $\begin{array}{c}\text { Molecular } \\
\text { weight } \\
\left(\mathrm{gmol}^{-1}\right)\end{array}$ & $\begin{array}{c}\text { Vapor } \\
\text { pressure } \\
(\mathrm{mmHg} \\
\left.@ 25^{\circ} \mathrm{C}\right)\end{array}$ & $\begin{array}{c}\text { Vapor } \\
\text { density } \\
\left(\mathrm{kg} / \mathrm{m}^{3}\right)\end{array}$ & $\begin{array}{c}\text { Viscosity } \\
\left(\mathrm{mPa} @ 20^{\circ}\right. \\
\mathrm{C})\end{array}$ & $\begin{array}{l}\text { Solubility } \\
\text { (in water) }\end{array}$ & $\begin{array}{c}\text { Boiling } \\
\text { point } \\
\left({ }^{\circ} \mathrm{C}\right)\end{array}$ & $\begin{array}{c}\text { Melting } \\
\text { point } \\
\left({ }^{\circ} \mathrm{C}\right)\end{array}$ & $\begin{array}{c}\text { Specific } \\
\text { gravity }\end{array}$ \\
\hline Hexane & $\mathrm{C}_{6} \mathrm{H}_{14}$ & 86.18 & 151 & 2.97 & 0.31 & Immicible & $62-69$ & -95 & 0.678 \\
\hline Ethanol & $\mathrm{C}_{2} \mathrm{H}_{5} \mathrm{OH}$ & 46.04 & 59.3 & 1.59 & 1.2 & Miscible & 78 & -114.1 & 0.79 \\
\hline Methanol & $\mathrm{CH}_{4} \mathrm{O}$ & 32.04 & 128 & 1.11 & 0.55 & Miscible & 64.7 & -98 & 0.791 \\
\hline Isopropanol & $\mathrm{C}_{3} \mathrm{H}_{8} \mathrm{O}$ & 60.09 & 33 & 2.1 & 2.27 & Miscible & 82 & -88 & 0.785 \\
\hline Acetone & $\mathrm{C}_{3} \mathrm{H}_{6} \mathrm{O}$ & 58.08 & 231 & 2 & 0.32 & soluble & 56.5 & -94 & 0.788 \\
\hline $\begin{array}{c}\text { Ethyl } \\
\text { acetate }\end{array}$ & $\mathrm{C}_{4} \mathrm{H}_{8} \mathrm{O}_{2}$ & 88.11 & 73 & 3.04 & 0.44 & $\begin{array}{c}\text { Slightly } \\
\text { soluble }\end{array}$ & 77 & -83 & 0.9 \\
\hline Acetonitrile & $\mathrm{C}_{2} \mathrm{H}_{3} \mathrm{~N}$ & 41.05 & 88.8 & 1.42 & 0.36 & soluble & 81.6 & -45 & 0.781 \\
\hline
\end{tabular}

*According to MSDS from Fisher Scientific International, Inc. 

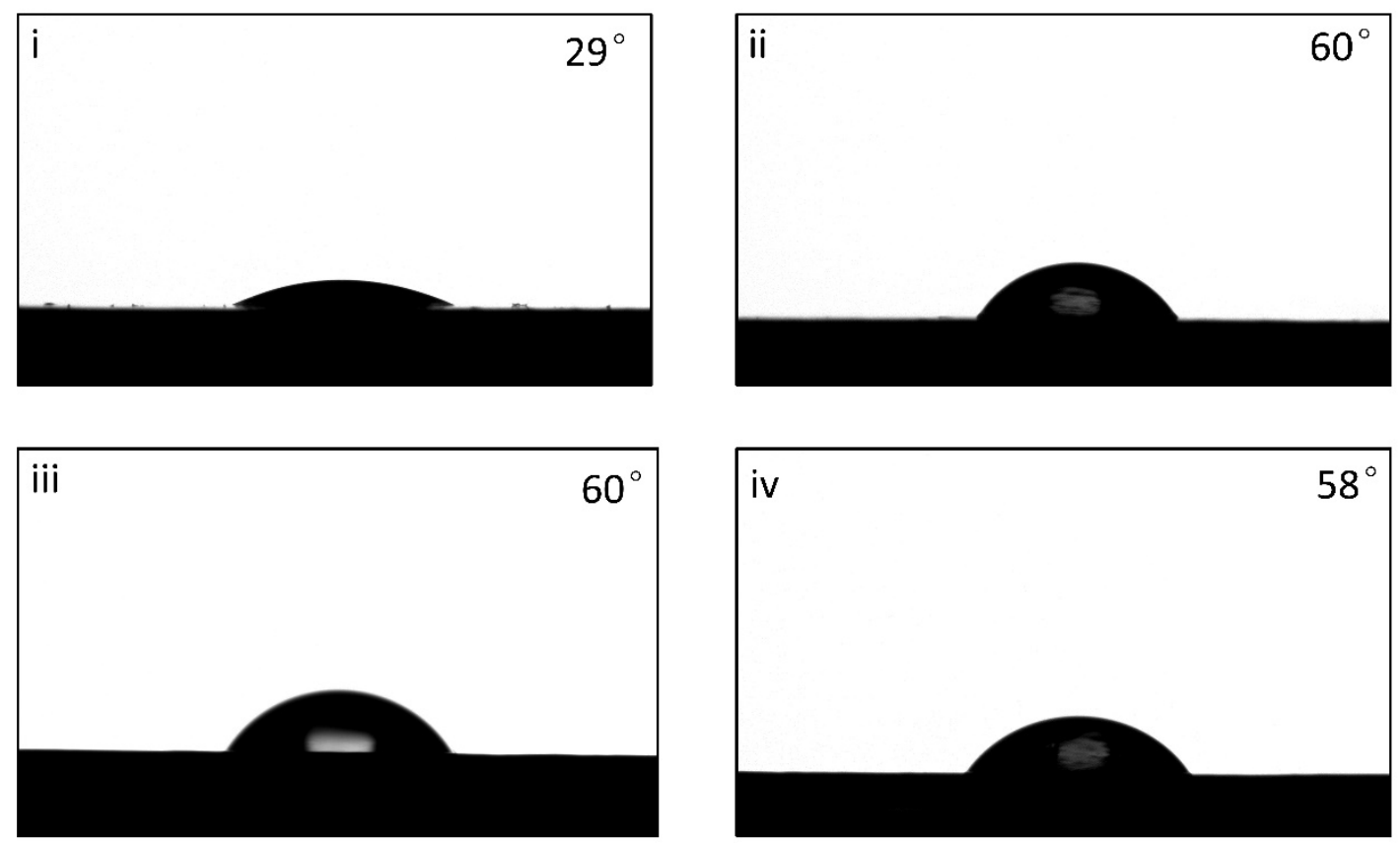

Figure S1. Contact angle measurement of (i) PAN porous support and (ii, iii, iv) polyamide-PAN membranes prepared with $0.05 \%, 0.1 \%$ and $0.15 \%$ TMC in decanoic acid as organic phase. 

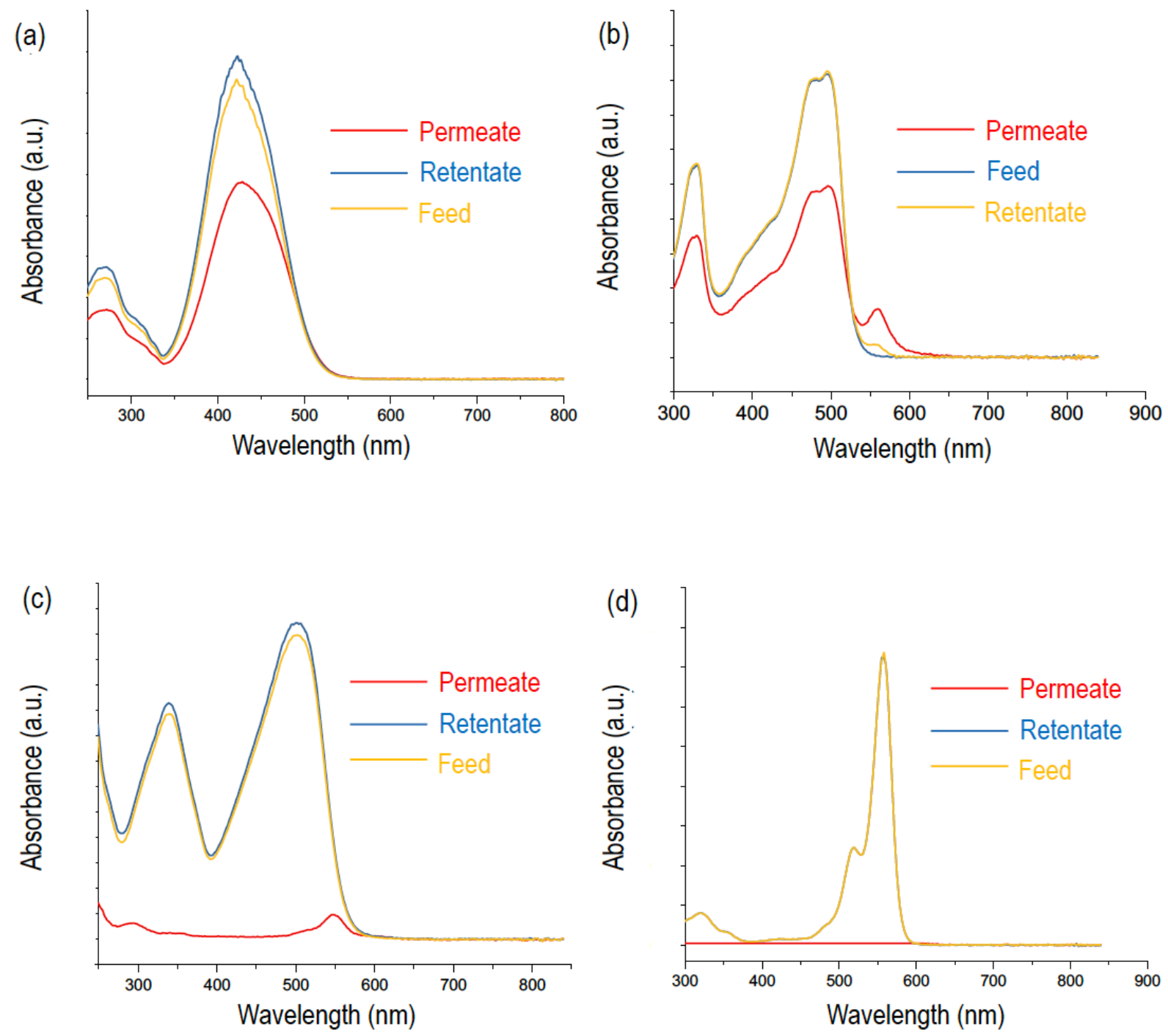

Figure S2. UV spectra of dye solutions in methanol, before and after filtration using a polyamide-PAN membrane (0.05\% TMC): (a) Methyl Orange, (b) Orange G, (c) Congo Red, and (d) Rose Bengal. 

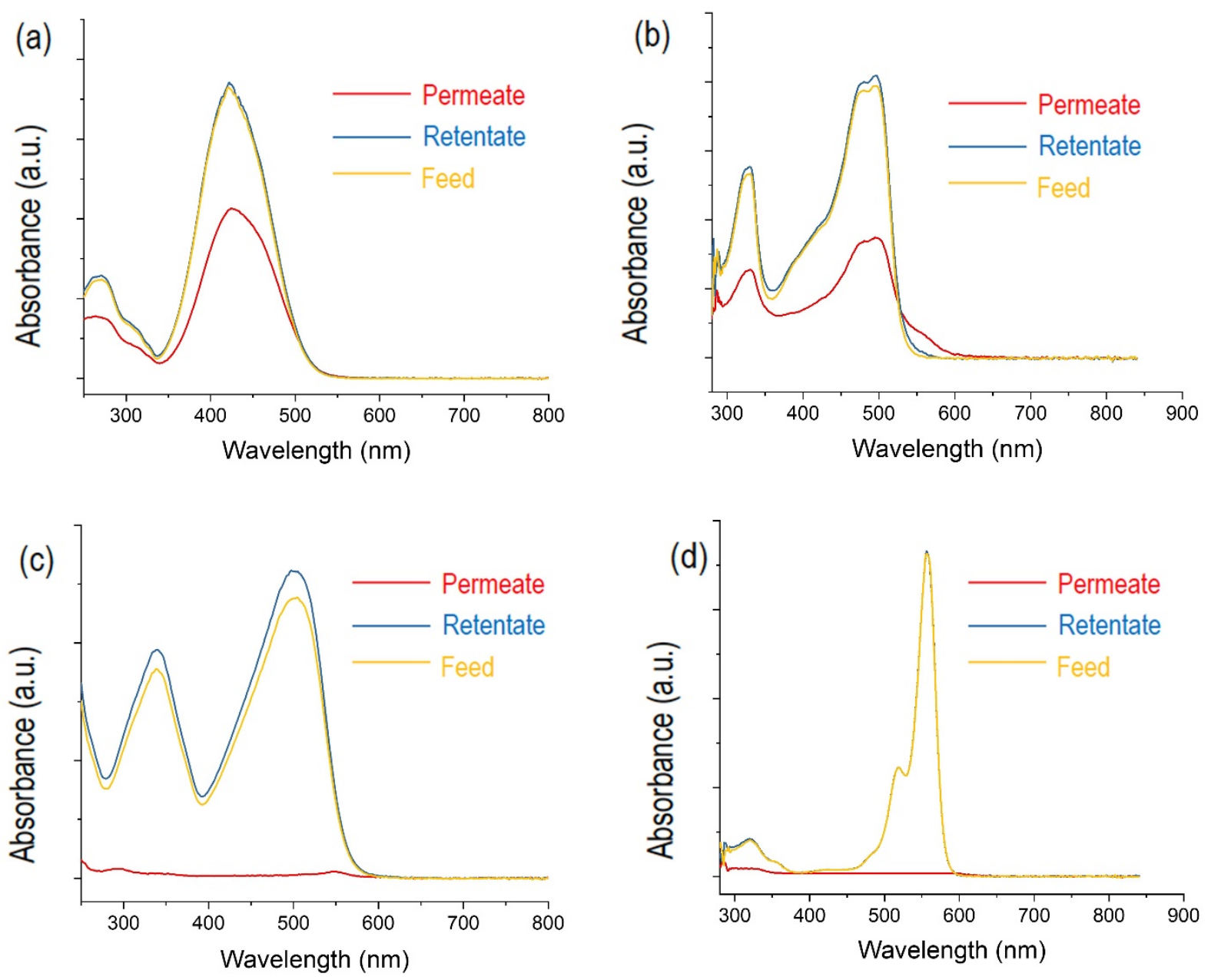

Figure S3. UV spectra of dye solutions in methanol, before and after filtration, using a polyamide-PAN membrane (0.1\% TMC): (a) Methyl Orange, (b) Orange G, (c) Congo Red, and (d) Rose Bengal. 
(a)
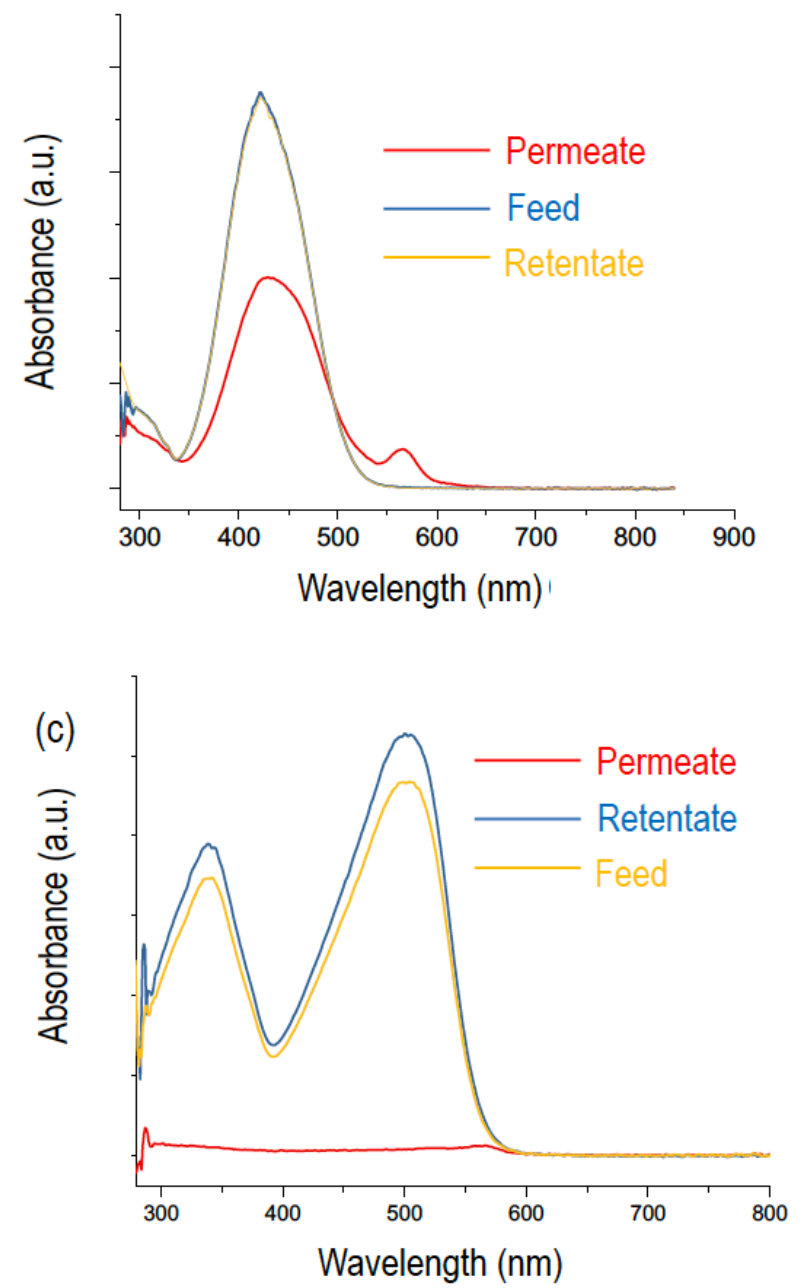

(b)
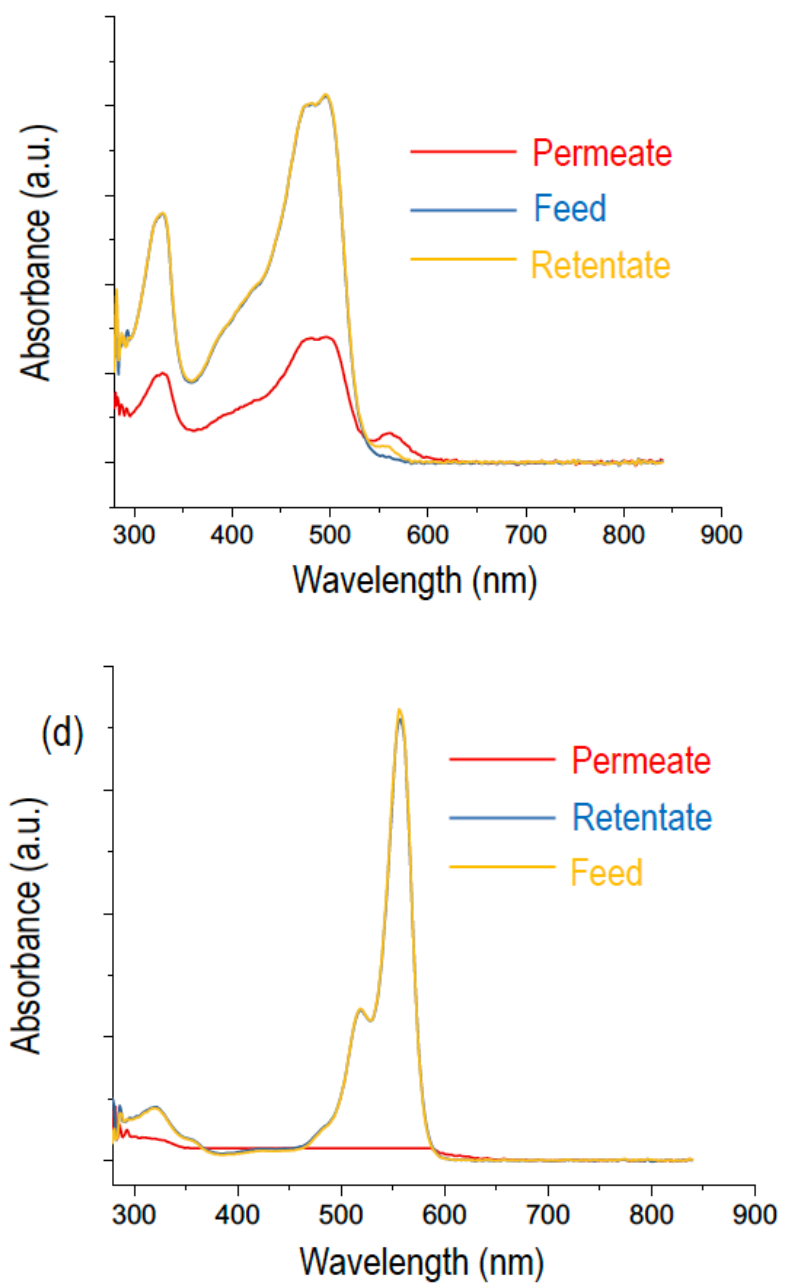

Figure S4. UV spectra of dye solutions in methanol, before and after filtration, using a polyamide-PAN membrane (0.15\% TMC): (a) Methyl Orange, (b) Orange G, (c) Congo Red, and (d) Rose Bengal. 
(a)

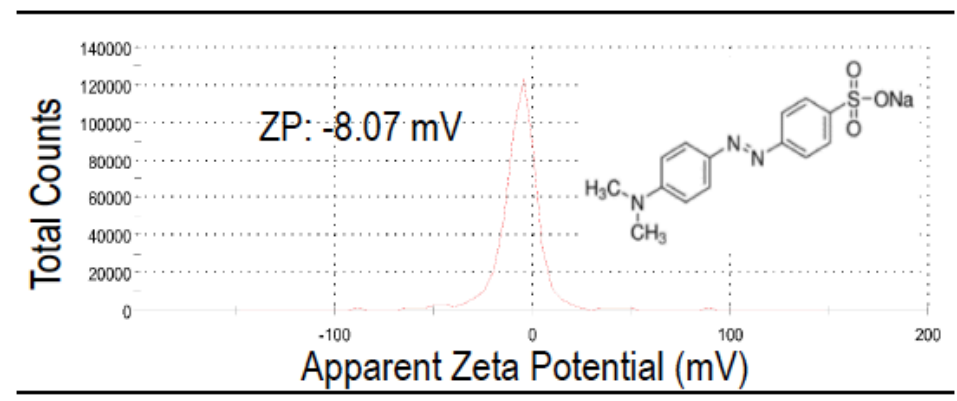

(b)

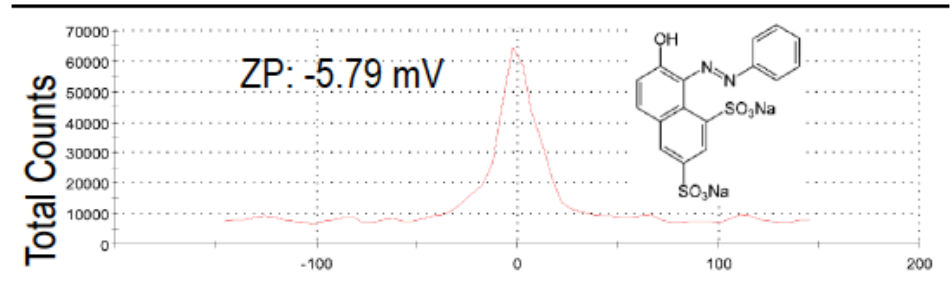

Apparent Zeta Potential (mV)

(c)

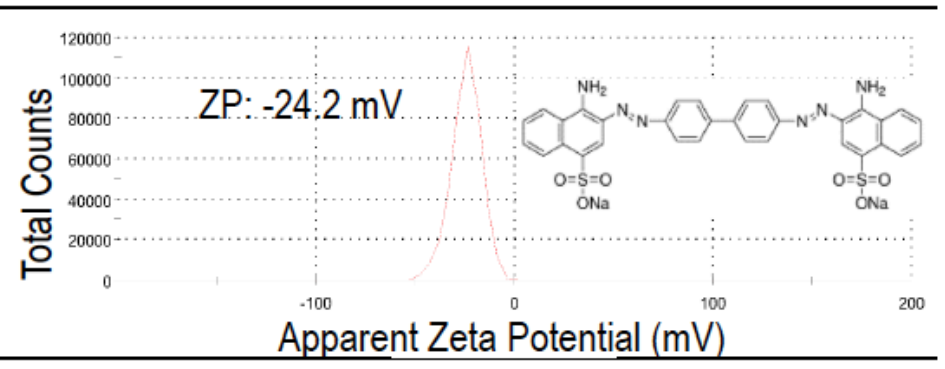

(d)

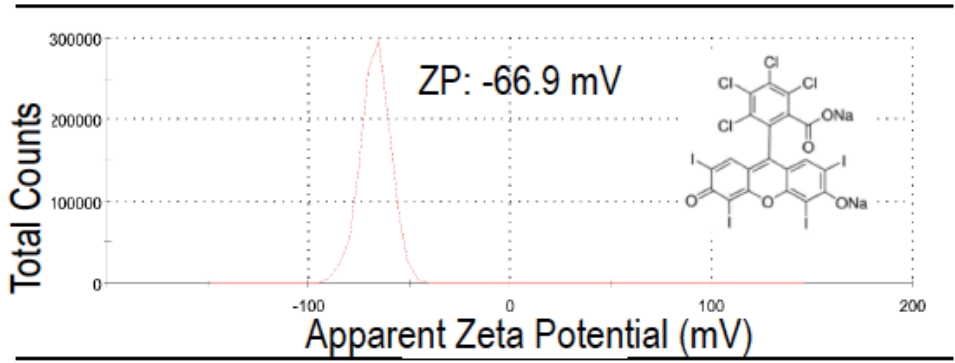

Figure S5. Zeta potential of (a) Methyl Orange (b) Orange G (c) Congo Red (d) Rose Bengal measured in solution in methanol. 

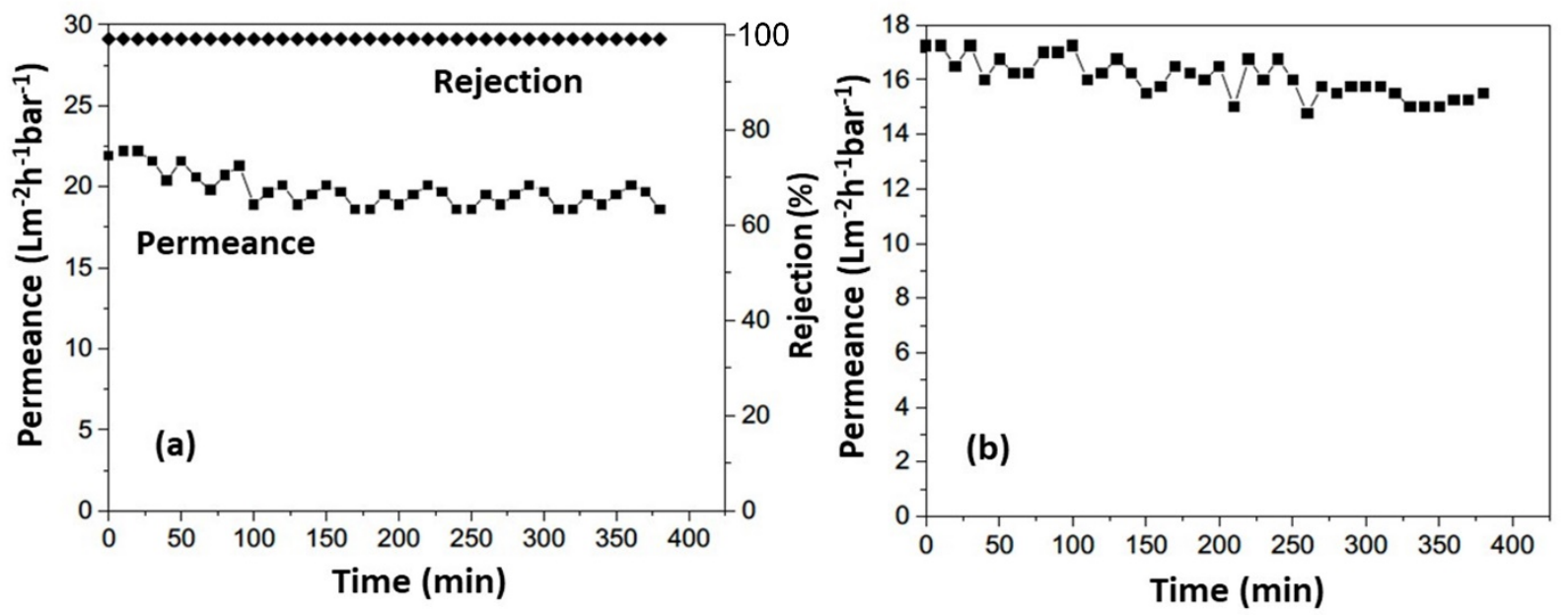

Figure S6. Long-term filtration tests: (a) methanol permeance and Congo Red rejection; (b) water permeance, using a polyamide-PAN membrane $(0.15 \% \mathrm{TMC})$. 
Table S3. Mean pore diameter of the polyamide-PAN membranes with different TMC concentrations.

\begin{tabular}{cc}
\hline & \\
TMC concentration (\%) & Mean pore diameter $(\mathrm{nm})$ \\
\hline $0.05 \%$ & 0.74 \\
$0.10 \%$ & 0.78 \\
$0.15 \%$ & 0.80 \\
\hline
\end{tabular}


Table S4. Effect of TMC concentrations to the membrane zeta potential.

\begin{tabular}{cc}
\hline $\begin{array}{c}\text { TMC concentration } \\
(\%)\end{array}$ & $\begin{array}{c}\text { Zeta potential } \\
(\mathrm{mV})\end{array}$ \\
\hline 0 & -49.4 \\
0.05 & 3.58 \\
0.1 & 10.2 \\
0.15 & -2.87 \\
\hline
\end{tabular}


Table S5. Solvent stability test for polyamide-PAN membranes $(0.1 \%$ TMC) in various solvents for 7 days.

\begin{tabular}{lc}
\hline & Weight loss $(\%)^{*}$ \\
\hline Hexane & $<0.2$ \\
Ethanol & $<0.2$ \\
Methanol & $<0.1$ \\
Isopropanol & $<0.1$ \\
Acetone & $<0.1$ \\
\hline
\end{tabular}

*Weight loss (\%) measured after immersing the membrane in various solvent for 7 days. 